Aus dem Institut für pathol. Anatomie des Obermedizinalrats Prof: Dr. Schmorl im Friedrich-Stadtkrankenhaus und aus der Abteilung für Syphilis und Eautkrankheiten des Oberarztes Dr. Werther in Dresden.

\title{
Zur Kenntnis der lokalen Veränderungen nach hypodermatischen Injektionen eines schwefelhaltigen Jodöls bei Syphilis.
}

\author{
Von \\ Dr. Tokujiro Suzuki, \\ Stabsarzt der Kaiserl. Japanischen Marine.
}

(Hiezu Taf. XII.)

Die lokalen Veränderungen nach intramuskulären Injektionen unlöslicher Quecksilbersalze sind von einigen Autoren im Tierexperimente und auch am menschlichen Muskelgewebe eingehend studiert worden. Welche Veränderungen jedoch die eingespritzten Jodpräparate im Gewebe erleiden, ist weniger bekannt, obwohl die hypodermatische Jodtherapie gegen die Syphilis gegenwärtig sehr verbreitet ist.

Von den in der Praxis am meisten gebräuchlichen Jodpräparaten ist vor allem das Jodipin zu nennen, ein von Winternitz (1) entdecktes und von Merck in Darmstadt in $10 \%$ igen und $25 \%$ igen starken Dosen aus Jod und Sesamöl in Verbindung mit Chlor hergestelltes Additionsprodukt. Bezüglich des subkutan angewandten Jodipins geben Klingmüller (2), Kindler (3), Sichatschew (4), Nobel (5), Wanke (6), Baum (7), Welander (8), Grouven (9), Möller (10), Feibes (11), Fr. Lesser (12) u. a. gleichmäßig an, daß in keinem Falle irgendwelche Störungen subjektiver oder objektiver Art beobachtet werden konnten und daß es ferner länger als Jodkali im Körper verbleibt. 
Dagegen zeigten sich in neuester Zeit doch unangenehme Resultate bei den im allgemeinen subkutan oder seltener intramuskulär vorgenommenen Injektionen von Jodipin bei Syphilis und anderen Krankheiten.

Mittler (13) hat jüngst in der Wiener Gesellschaft für innere Medizin einen Pat. besprochen, der seit 3 Jahren an schwerer Lues litt, eine Unmenge Jodipiniujektionen und zwar viele derselben in die Muskulatur der beiden Oberschenkel bekam. Er hat nach der Untersuchung mit Röntgenstrahlen eigentümliche Gebilde, teils in Tropfenform, teils in mehr rundlicher Körnerform gefunden und führt diesen Befund auf eine mit Verkalkung oder Verknöcherung einhergehende Erkrankung der Fascien und interstitiellen Bindegewebssepten zurück.

Sandow (14) hat bei einem Pat., der wegen eines hartnäckigen Rheumatismus eine $25 \%$ ige Jodipininjektion in dem Arm bekommen hatte, 2 Monate später außer einem Knochenabszeß im Oberarm - auf den wahrscheinlich die schon in der Jugend des Kranken periodisch aufgetretenen Schmerzen im Arme zurückzuführen waren - unter den Röntgenstrahlen undurchlässige dunkle Streifen im Vorderarm beobachtet. Er bemerkte nach einer Probeinzision in den Vorderarm, daß die zwischen Vorderarm-Fascie und Muskulatur gelegene dünne Fettschicht ein auffallend zitronengelbes Aussehen zeigte. Das exstirpierte Fettgewebe ergab sehr deutliche Jodreaktion und auch die Muskelstücke ent. hielten Spuren von Jod; ferner fiel auch die Urinuntersuchung positiv aus und zwar war noch 5 Monate nach der Vornahme der Injektionen deutlich Jod im Harn nachzuweisen. Er glaubt, daß der betreffende Arzt offenbar mit der Nadel die Vorderarmfascie durchbohrt und das Jodipin in den subfascialen Raum gespritzt habe, wo es sich innerbalb der Längsfächer ausgebreitet und gesenkt hatte. Wir dürfen schon nach den Sehw aldschen (15) Untersuchungen annehmen, daß die Halogene im reinen Zustande ebenso wie die chemischen Verbindungen derselben für die Röntgenstrahlen in hohem Maße undurchlässig sind; daß auch das Jodipin als eine chemische Verbindung des Chlorjods mittelst der Röntgenstrahlen im Körper nachweisbar ist, konnte $S a n d o w$ in seinem Falle in ausgezeichneter Weise bestätigen.

Leider aber sagen alle diese Autoren nichts ïber die wichtigen histologischen Erscheinungen bei der Anwendung des Jodipins und über die lokale Wirkung auf das Gewebe, in welches injiziert wurde.

Das Übergehen dieses Punktes ist erklärlich, wenn man bedenkt, mit welchen Schwierigkeiten die Beschaffung des solchen histologischen Forschungen als Unterlage dienenden Materials verbunden ist. 
Im Sommer 1903 hatte ich Gelegenheit, die Frage der Wirkung eines $\mathrm{schwefelhaltigen} \mathrm{Jodöles} \mathrm{eingehend} \mathrm{zu} \mathrm{stu-}$ dieren. Es war im Friedrichstädter Krankenhause in Dresden ein Mann wegen Syphilis mit hypodermatischen Injektionen von einem schwefelhaltigen Jodöl, das eine Farbfabrik zum Zwecke therapeutischer Prüfung zur Verfügung gestellt hatte, behandelt worden. Der Mann starb 5 Monate nach der letzten Injektion infolge einer interkurrenten Appendicitis. Die von mir vorgenommene histologische Untersuchung der Leiche ergab, wie ich glaube, interessante Resultate in Bezug auf die örtliche Wirkung des Jodöls, und es dürfte nicht unnütz sein, die Ergebnisse meiner Untersuchungen zu veröffentlichen und sie mit den von Jadassohn (16) und Fr. Lesser (17) am Tiermuskel, Wolters (18) und Pezzoli (19) am Menschenmuskel mit Hydrargyrum salicylicum und Jullien (20), Allgeyer (21), A udry (22) u. a. mit Kalomeleinspritzungen am menschlichen Muskel erhaltenen zu vergleichen.

Bevor ich zur Beschreibung meiner Untersuchung übergehe, muß ich einige Worte über das zur Verwendung gekommene Jodöl sagen, das aus mancherlei Gründen bis jetzt nicht in den Handel gebracht worden ist. Ich lasse die Hersteller des Präparates selbst sprechen:

"Es ist ein Jodsesamöl von gelber Farbe, schwerer als Wasser, von indifferentem Ölgeschmacke. Es sind 2 Sorten hergestellt. Die eine enthält genau $10 \%$ organisch gebundenes Jod, welches sich nicht direkt nachweisen läßt und außerdem noch $1.6 \%$ unoxydierten Schwefel; die andere neben $25 \%$ Jod $2.5 \%$ unoxydierten Schwefel. Weder durch Schütteln mit Wasser, noch mit stark verdünnten Alkalien oder Säuren werden merkbare Mengen von Jod abgegeben. Trotzdem wird das Jod im Organismus leicht abgespalten.

Der reiche Jodgehalt dieses Medikamentes läßt sich auf einfache Weise leicht dadurch nachweisen, daß man das Öl im trockenen Reagensglase über freier Flamme vorsichtig erwärmt. Nach kurzem Erwärmen entweicht das Jod in großer Menge in Form des charakteristischen violetten Dampfes, während sich das Öl braun bis schwarz färbt.

Es ist also ein dem Jodipin analoges Präparat, von welchem es sich durch den schon angegebenen Schwefelgehalt unterscheidet und es dürften so ziemlich die gleichen Indikationen in Betracht kommen, wie für das Jodipin und die bekannten Jodsalze.

Im Gegensatz zu den Jodalkalien erfolgt die Jodabspaltung langsamer, niemals treten so hohe Jodalkalienmengen im Blute auf und die Arch. f. Dermat. u. Syph. Bd. IXXXV. 
Ausscheidungszeit ist verlängert. In allen diesen Beziehungen überragt dieses Präparat das Jodipin. Ausgeschieden werden durchschnittlich von Jodkali $78-80 \%$, von Jodipin $58.5 \%$, von unserem Präparat $45.1 \%$. Spaltung erfolgt nur zum geringsten Teil im Dünndarm, vornehmlich im Blut, wo es fein verstäubt ankommt. Es wird als Körperfett angesetzt und in den Ausscheidungsdrüsen der Fette (Milchdräsen, Hautdrüsen) ausgeschieden. Die Ausscheidungsdauer beträgt nach $1 \mathrm{~g}$ Jod für Jodkali 42-60 Stunden, Jodipin $4^{1 / 2}-5$ Tage, bei unserem Präparat $6^{1 /} / 2-8$ Tage; bei Einnehmen von $25 \mathrm{~g}$ Jodfett $3 \mathrm{mal}$ täglich während dreier aufeinanderfolgender Tage: für Jodipin 61/2 Tage, für unser Präparat 12 Tage, von der Darreichung der letzten Dosis an gerechnet.

Der Schwefel in diesem Präparat spielt nur die Rolle eines Jodüberträgers. Merck benutzt bei seinem Präparat hierzu Chlor; das Jodipin ist demgemäß chlorhaltig. Zu irgend welchen Komplikationen bei merkurialisierten Kranken dürfte der S-Gehalt indessen nicht Anlaß geben, da der Schwefel sehr fest gebunden ist. Diesbezügliche Versuche an mit $\mathrm{Hg}$ behandelten Tieren lassen sich leider nicht anstellen; sie würden eine solche Kur nicht aushalten.

Dieses für sublutane Injektionen bestimmte Präparat ist im Gegensatz zum Jodipin, in welchem wir zahlreicbe Luftkeime aufgefunden haben, steril. Der Einspritzung dieses sterilen Präparates steht nichts im Wege, dieselbe wird ohne $Z$ weifel gut vertragen."

In der Abteilung für Hatt- und Geschlechtskranke des Friedrichstädter Krankenhauses wurden von Juli 1902 bis April 1903 mit sublkutanen Einspritzungen von schwefelhaltigem Jodöl 6 Patienten und durch innerlichen Gebrauch desselben Mittels ein Patient behandelt. Alle Pat. litten an tertiärer Lnes; dem letztgenannten war der Geschmack des Jodöls widerlich, trotzdem nahm er in elf Tagen $120 \mathrm{~cm}^{3} \quad 10 \%$ iges und $50 \mathrm{~cm}^{3} 25 \%$ iges Jodöl ein und zwar mit gutem Erfolg. An 5 Pat. unter 6 , welchen jeden zweiten oder dritten Tag $10 \mathrm{~cm}^{3} \quad 10 \%$ iges oder $3 \mathrm{~cm}^{3} 25 \%$ iges Jodöl tief subkutan injiziert wurde, bemerkten wir keine Zeichen von Jodismus. Sie vertrugen die Injektionen ausgezeichnet, an der Injektionsstelle ließen sich keine anhaltenden Indurationen wahrnehmen; die Pat. wurden sämtlich nach $25-40$ Einspritzungen geheilt entlassen.

Ich gehe jetzt zu dem Falle über, der mir das Material zu meiner Arbeit geliefert hat. Aus der Krankengeschichte sei hier nur kurz folgendes mitgeteilt:

W. Z., 55 Jahre alt, Handarbeiter, aufgenommen am 31. Dez. 1902 auf die Abteilung des Oberarztes Dr. Werther im Stadtkrankenbaus, 
infizierte sich vor 10 Jahren, war 1897 das erste Mal wegen Syphilis in demselben Krankenhaus, später noch verschiedene Male; hat 4 Schmierkuren durchgemacht und Jodkali genommen. Seit 2 Monaten litt Patient an Heiserkeit und Atemnot, die sich namentlich Nachts oft beängstigend steigerte. Das Schlucken ging schlecht.

Status: Mittelkräftiger Mann, etwas abgemagert, Haut- und Schleimbäute blaß. An den Extremitäten alte von Hautgeschwüren herrührende Narben. Kurzatmig, ängstlicher Gesichtsausdruck. An den Zwischenrippenräumen inspiratorische Einziehung. Stenosenatmen über den Lungen, nebenbei Bronchitis. Milzrand palpabel. Urin: ohne Zucker, ohne Eiweiß. Sarcocele syphilitica. Organe im übrigen o. B.

Laryngoskopischer Befund: Verdickung der Epiglottis, dieselbe ist rinnenförmig. Vom linken Aryknorpel hängt ein längs-ovaler Fleischwulst in das Lumen des Kehlkopfes hinein, dasselbe zum Teil verdeckend. Die Taschenbänder sind dunkelrot, stark geschwollen. Beim Atmen wie Phonieren totale Ankylose der Gelenke, alles - soweit übersehbar - ist regungslos. Hochgradige Stenose. Therapie: Injektionen unter die Haut der Pektoralisgegend von $25 \%$ schwefelhaltigem Jodöl, vom 2. bis 19. Januar $46 \mathrm{~cm}^{3}$ in 11 Injektionen. Das Jod machte keine Akne, keinen Schnupfen; jedoch bildeten sich Indurationen an den Injektionsstellen, welche auf Betasten schmerzhaft waren und schlieBlich eine harte, tiefe Platte bildeten.

Da Pat. bis 16./I. außerdem $3 \mathrm{~kg}$ an Körpergewicht abnahm, wurde diese Therapie ausgesetzt, dafür $2 \mathrm{~g}$ Joduatrium pro die gegeben und Inunktionen à 3.0 gemacht.

Am 8. März 1903 befand Patient sich merklich wohl, ging längere Zeit im Garten spazieren, hatte ausgezeichneten Appetit. Nur noch bei größeren Anstrengungen mäßiger Stridor. Der vom linken Aryknorpel ausgehende Tumor war bedeutend kleiner geworden. Die bisher durch Schleim- und Eiterauflagerung gedeckte Keblkopfschleimhaut war jetzt reiner und blasser.

Die Kehlkopfstenose wurde durch Herrn Dr. Max Mann vorsichtig mit Zinnbougies erweitert.

Bis 28. März sechsmal bougiert.

5. Mai. Im Anschluß an eine Bougierung, die zum ersten Male bis in die Trachea ging, starke Dyspnoe (wahrscheinlich infolge von Ödem). Patient wurde deshalb auf die chirurgische Abteilung verlegt, nachdem er i. S. $46 \mathrm{~cm}^{3}$ Jodipin $25 \%, 200 \mathrm{~g}$ NaJ und 24 Einreibungen bekommen und an Körpergewicht $10^{\circ} 6 \mathrm{~kg}$ zugenommen hatte. Die Dyspnoe ging unter Morphium zurück. Patient wurde am 16. Mai 1903 entlassen. Am 30. Mai $1903 \mathrm{kam}$ er sua sponte wiedex, wurde tracheotomiert; nach anfänglichem Wohlbefinden erfolgte am 24. Juni 1903 der Exitus durch Peritonitis infolge einer interkurrenten Appendicitis.

Sektionsdiagnose: Eitrige Peritonitis. Phlegmone in der Umgebung des Wurmfortsatzes. Ausgedehnte Narbenbildung im Kehlkopf. Tracheotomiewunde. Alte verkreidete Herde in der Lungenspitze. Lungen. 
ödem. Aspiration von Mageninhalt. Arteriosklerose. Fettentartung des Herzfleisches. Milztumor, Ikterische Fettleber. Schwielige Orchitis links. Beginnende Pyelitis.

Im subkutanen Fettgewebe der Brust finden sich zahlreicheHerde, in deren Bereich das Bindegewebe schwielig verdickt ist: beim Einschneiden dieser Herde quillt eine ölige, olivengrüngefärbte Flüssigkeitineinzelnen Tropfen and Tröpfchen hervor. Einiger solcher Schwielenherde befinden sich auch in der Substanz des muscul. pectoral. major.

In einer Axillard rüse ein erbsengroßer, gelblicher Käseherd.

Bei der Sektion fanden sich, außer luetischer Narbenbildung im Kehlkopf, keinerlei mit Lues in Zusammenhang stehende Erscheinungen in den inneren Organen. Mein Hauptinteresse war auf das subkutane Gewebe der Brustwand gerichtet, in welches die Injektionsmassen deponiert waren. Obermedizinalrat Prof. Dr. Schmorl stellte mir in der liebenswürdigsten Weise die uns interessierenden, von der Leiche entnommenen Subkutangewebe aus verschiedenen Brustwandstellen zur Verfügung, wofir ich mir auch an dieser Stelle meinen besten Dank auszusprechen eriaube.

Nach Abpräparierung der Haut fanden sich an der Stelle, wo die tiefen Injektionen gemacht worden waren, tief bis in Mitte des Muskelgewebes hinein zablreiche parallel mit der Richtung der Muskelbündel verlaufende zirka 10-15 $\mathrm{cm}$ lange derbe Stränge, bald breiter, bald schmäler, meist von der Dicke eines Fingers. Beim Durchschneiden trat aus zahlreichen, kleinen, rundlichen oder ovalen, einer Honigwabe ähnlichen Hoblräumen etwa vom Durchmesser eines Stecknadelkopfes bis einer Frbse eine ölige, olivengrüngefärbte Flüssigkeit in einzelnen Tropfen and Tröpfehen hervor. Das die Hohlräume umgebende Gewebe stellte ein graugelbliches, festes, schwielich verdicktes Bindegewebe dar, das sich von dem umliegenden gelben normalen subkutanen Fettgewebe nnd der rotbraunen normalen Muskulatur deutlich abhob. Es fanden sich einige solcher Schwielenherde auch in der Substanz des Muscul. pectoral. major. Wie viele solcher Herde vorlagen, wurde nicht festgestellt, doch glauben wir nicht fehl zu gehen, wenn wir annehmen, daß jeder Injektion ein solcher entsprach und $\mathrm{da} ß$ wir es somit zu tun hatten mit den Residuen der Jodöleinspritzungen. In Übereinstimmung hiermit ergab eine chemische Untersuchung dieses Inhalts - Behandlung mit Ätznatron, Lösung in Wasser, Filtration, Ansäuerung mit rauchender Salpetersäure und Ausschütteln mit Chloroform starke Violettfärbung -- sehr dentliche Jodreaktion. Zum Zwecke der histologischen Untersuchung wurden die verschiedenen Herde verwendet, in welchen, wie gesagt, in der Zeit vom 2. bis 19. Januar 11 Injektionen, die letzte also 5 Monate vor dem Tode, vorgenommen worden waren. Wir präparierten den ganzen oben beschriebenen Strang heraus, zerschnitten ihn in würfelförmige Stücke, in Längs- und Querschnitte in der Mitte und am Ende des Stranges, welche 
wir zur Konservierung in Formalin legten, um den Öl- und Blutgehalt nicht zu zerstören. Nachdem die Stücke durch mehrstündliches Auswässeru vom Formalin befreit waren, wurden sie mit Beckerschem Koblensäuregefriermaikrotom in $10-15 \mu$ dünne Schnitte zerlegt, dann nach verschiedenen Methoden gefärbt. Zar Feststellung des Öls benutzten wir die Färbung mit Sudan III (in 70\% iger Alkohollösung nach Daddi und Mi chaelis) und gleichzeitig zur Kernfärbung Hämatoxylin nach Delafield, worauf die Schnitte in Glyzerin eingelegt wurden. Zum Nachweis der elastischen Fasern kam die Weigertsche Methode zur Anwendung.

An den so behandelten Präparaten traten die Gewebselemente, auf deren Darstellung es vorwiegend ankam, außerordentlich dentlich hervor. Außerdem wurden zur genaueren Untersuchung der feinen Zellstruktion die Stücke in Paraffin eingebettet, geschnitten und gefärbt und zwar nach verschiedenen Kernfärbungsmethoden. Zum Nachweis der Mastzellen benutzten wir das bekannte Unnasche polychrome Methylenblan und zur Entfärbung die Unnasche Glyzerinäthermischung oder kurzes Eintauchen in $1 / 2 \%$ ige Essigsäure und Übertragen in konzentrierte, zur Hälfte mit Wasser verdünnte Alaunlösung.

\section{Mikroskopische Untersuchung.}

Die Präparate, die wir aus dem oben erwähnten Strange genommen hatten, boten ein etwas verschiedenes Bild dar, je nachdem sie von den Längsschnitten oder Querschnitten, aus der Mitte oder von den beiden Enden des Stranges in der Gegend, wo das injizierte öl eingedrungen war, herstammten.

Schnitte durch die beiden Enden des geschilderten Stranges senkrecht zu ihrer Längsrichtung und durch die benachbarten Gewebe, subkutanes Fettgewebe und Muskulatur, zeigten, daß im Zentrum reichliches, neugebildetes, gefäßreiches Bindegewebe (Granulationsgewebe) vorhanden war, das sich peripherwärts in das benachbarte Fettgewebe und die angrenzende Muskulatur hinein fortsetzte.

In den Querschnitten des mittleren Teiles des Stranges fiel eine große Zahl von Hohlräum en auf, die zum Teil schon mit freiem Auge zu erkennen waren. Einen Inhalt konnten wir in den Hohlräumen an den unmittelbar nach der Sektion gewonnenen Gefrierschnitten nicht wahrnehmen, erst nach Fixierung von Formalin und nach Färbung mit Sudan zeigte sich, daß in diesen Hohlräumen Öl vorhanden war. Das Öl füllte den Hohlraum entweder ganz aus oder lag der Innenfläche bloß als eine mehr oder minder schmale Schicht an; außerdem fanden sich in der Wand der Hohlräume isolierte größere und kleinere Öltröpfchen. Die Größe dieser Hohlräume war sehr verschieden; die kleineren erreichten kaum die Größe einer normalen Fettzelle und waren fast durchweg rund oder 
oval; man erkannte an ihrer Peripherie eine einfache Lage von schmalen, länglichen Zellen etwa rom Aussehen der Endothelien, auch kamen häufig Auskleidungen vor, die aus protoplasmareichen Gebilden bestanden und zum größten Teil keine deutlichen Zellgrenzen erkennen ließen, sondern konfiuierende Zellmassen mit zahlreichen, oft zu Haufen angeordneten, sehr dunklen Kernen darstellten rom bekannten Aussehen der Fremdkörper-Riesenzellen. Die Hohlräume wurden teils nur von Riesenzellen allein ausgekleidet, teils aber waren zwischen diesen auch einige schmale Z ellen eingelagert, in denen nur ein Kern sichtbar war. In den meisten Hohlräumen erschien der Zellbelag deutlich abgeplattet und machte den Eindruck, als wäre er durch den Inbalt des Hohlraumes stark komprimiert worden. Sowohl in den schmalen. endothelartigen Zellen, als auch ganz besonders in den protoplasmareichen Zellen und Riesenzellen fanden sich sehr feine Fettröpfchen. Die größeren Hohlräume waren mitunter von einer aus konzentrisch angeordneten Bindegewebsfasern und Spindelzellen bestehenden Gewebsschicht umsäumt. wodurch diese Hohlräume eine gewisse Ähnlichkeit mit Gefäßquerschnitten erbielten, die noch dadurch erhöht wurde, daß sie an ihrer inneren Wandung mit flachen endothelartigen Zellen ausgekleidet waren (vergl. Taf. XII, Fig. $1 A$ ). Daß es sich aber nicht etwa um Gefäße, in die Öltropfen eingedrungen waren, handelte, zeigte ohne weiteres die Weigertsche Elastinfärbung, die nirgends die für die Gefäßwandung charakteristische Anordnung der elastischen Fasern nachweisen ließ. An einzelnen Stellen standen die Hohlräume so nahe auseinander, daß das Gewebe ein feinschwammiges Aussehen gewann (vgl. Fig. $1 B$ ).

Neben den kleineren Hohlräumen traf man sehr häufig auch auf große Spalten, die den makroskopisch erkennbaren, mit Öl gefüllten Hohlräumen entsprachen. Diese Spalten, aus denen infolge der Präparation das Öl ausgeflossen war, wurden von einem sehr zellenreichen Gewebe umgrenzt, zeigten aber nicht, wie die kleinen Hohlräume eine Auskleidung mit endothelartigen Zellen, ihre Wand bestand vielmehr größtenteils aus vielkernigen großen Riesenzellen, welche mitunter die eigentümlichsten Formen zeigten, die sich offenbar daraus erklärten, daß sie sich der Obertläche größerer, in den Hohlräumen liegender, aber bei der Präparation ausgeflossener Öltropfen angepaßt hatten. Auch in dem die Hohlräume einschließenden Granulationsgewebe fanden sich vielfach Riesenzellen, die größere und kleinere Öltropfen umschlossen.

Was nun das z ellig e Infil trat des Bindegewebes, welches wir in allen Schnitten des Stranges fanden, betrifft, so bestand dasselbe zumeist aus mononucleären Rundzellen, fixen Binde- 
gewebszellen, großen Leukocyten, nur zum kleineren Teil aus polynucleären Leukocyten, auch Plasmazellen und Mastzellen und zeigte entweder eine mehr diffuse oder wenig herdförmige Anordnung, doch ließen sich einkernige Fibroblasten oder auch Fremdkörperriesenzellen, welche denen in den Hohlraumwänden mehr oder weniger ähnlich und teils einzeln, teils in kleinen Gruppen vorkamen, meist ohne Schwierigkeit innerhalb des Herdes nachweisen (vgl. Fig. 1, D). Wenn diese Riesenzellen in verhältnismäßig weniger diffusen Zelleninfiltrationsherden lagen, so hatten sie mit jenen unten zu erwähnenden Muskelfasern, an denen Kernwucherung des Sarkolemm oder benachbarter Bindegewebszellen aufgetreten war, einige Ähnlichkeit, doch ließen sich letztere zumeist dadurch unterscheiden, daß sie genau die Form der Dimension von quer- oder schräggeschnittenen Muskelfasern zeigten. In diesen Infiltrationsstellen befanden sich mehrere kleinere oder größere Öltröpfchen, welche von den Leukocyten und den in Proliferation befindlichen protoplasmareichen Gewebszellen und vielkernigen Riesenzellen umfaßt waren und ebenso hatten die daselbst befindlichen Zellen meist äußerst fein ovale Fettröpfchen in ihrem Protoplasmaleib aufgenommen, zeigten ferner trübe Schwellung, körnigen Zerfall und vacuoläre Degeneration, wie ich sie auch in den Auskleidungszellen der Hohlräume und im Gewebe der Hohlraumwände und Umgebung derselben gefunden habe (vgl. Fig. 2.)

In der Mitte der starken Infiltrationsherde bemerkte man einige hellere und diffus homogen veränderte Bezirke, welche die Kernfärbung nicht angenommen hatten und an diesen Stellen fanden sich auch fädige Massen, in denen man noch einige blaß gefärbte isolierte Kerne von aufgequollenen Zellen erkennen konnte. Hier ist der degenerative Prozeß offenbar intensiver gewesen, indem es hier zu einer Nekrose der Zellen kam, welche wohl unter dem Einfluß des intensiv reizenden Jodöls entstand. Solche Herde mit nekrotischem Zentrum gleichen dann auffällig miliaren Gummata oder Tuberkeln, da ja in ihrer Peripherie auch Rundzellen, Epitheloidzellen ähnliche Fibroblasten und Riesenzellen vorhanden sind. Daß es sich aber nur um Pseudotuberkel handelt, geht, abgesehen ron den später zu erwähnenden Tierversuchen auch daraus hervor, daß die Riesenzellen und Fibroblasten reichlich Fettröpfchen, sowie Drusen von Fettsäurekristallen (in den Riesenzellen) enthielten (s. Fig. 2).

In stärkeren Entzündungsherden fanden sich bei der entsprechenden Färbung keine elastischen Fasern, nur in vereinzelten kleinen Herden wurden kurze Bruchstücke von elastischen Fasern gefunden. 
Das zwischen den Hohlräumen befindliche Gewebe stellte entweder ein mehr oder weniger stark zellig infiltriertes urid gefäßreiches Bindegewebe dar, oder es bestand aus einzelnen oder gruppenweise angeordneten Muskelfasern. Letztere zeigten verschiedene Grade von Atrophie, mitunter ganz enorme Grade, wobei sich ihre Kerne entweder normal verhielten, oder deutliche Wucherungsvorgänge zeigten, indem dann die Oberfläche solcher Muskelfasern von einer großen Zahl dicht stehender Kerne bedeckt erschien oder indem sie sich selbst verschmälert und abgeplattet, aber ohne Zerfallerscheinungen zeigten.

Außerdem fanden sich in anderen Präparaten, die aus den Längsschnitten der mittleren Partien des Stranges stammten, neben den obigen Erscheinungen noch andere $\mathrm{Mu}$ s $\mathrm{k}$ e $\mathrm{l}$ fa s e r veränderung en. Man bemerkte, daß hier die Muskelfasern kernlos geworden und homogen waren, ferner waren sie oft abgestorben, hyalin geronnen, blabrötlich, mattglänzend bereits im scholligen Zerfall begriffen (wach sartige Degeneration.) Sie erschienen schon makroskopisch im Präparat als hellere Stelle; mikroskopisch bemerkte man dementsprechend bei Hämatoxylintärbung, daß solche Fasern einen diffus rötlichbraunen Ton annahmen, während bei Anwendung von polychromen Methylenblau und nachfolgender Glyzerinätherbehandlung ein großer Teil der Fasern eine blaßgrünliche Farbe behielt. Man fand zwischen normalen Muskelfasern eine oder mehrere degenerierte, in Schollen zerfallene Muskelfasern von wechselnder Mächtigkeit und das zwischen diesen nekrotischen wachsartig degenerierten Muskelfasern gelegene Bindegewebe bildete so überall ein zellig-fibrinöses Gewebsinfiltrat zum Teil mit ge ronnenem Exsudat. An der Peripherie der nekrotischen Muskelpartien bemerkte man einige Muskelfasern, welche durch ihre oben erwähnte Kernwucherungen und gleichzeitige Veränderung ihres Farbetons ganz ähnlich wie die in den Hohlraumwänden gelegenen Fremdkörper-Riesenzellen aussàen. Diese Kernwucherungen waren in anderen Fällen nicht auf der Wucherung der Sarkolemmkerne zurückzuführen, sondern wahr. scheinlich Abkömmlinge der benachbarten Bindegewebszellen. die nach Art der sogenannten Fremdkörper-Riesenzellen bei der Resorption der nekrotischen Muskelfasern mitwirkten, also sie teilweise oder ganz umschlossen, wie ja auch z. B. Litten (23) bei der Resorption von Muskelfasern Riesenzellen gesehen und später auch P ezzoli (19) beobachtet hat. Hierbei konnten die Riesenzellen schon einen beträchtlichen Teil des Sarkolemmschlauches einnehmen, während die von ihnen eingeschlossene kontraktile, nekrotische Substanz auf einen schmalen Rest vermindert erschien. 
Die überall im Gewebe reichlich gefundenen Blutgefäße wiesen eine bindegewebige Verdickung ihrer Wandung und nicht selten eine geringe Wucherung ihrer Endothelien auf (vergl. Fig 1, E).

Außerdem bemerkten wir überall im Gewebe und besonders in den erwähnten, an den Hohlraumwänden gefundenen Riesenzellen und anderen Gewebszellen, welche in der Umgebung der Infiltrationsstellen lagen und nicht selten in den Zellen, welche sich in den stark entwickelten Bindegewebszïgen fanden und auch in einigen Muskelfasern, an denen Kernwucherung aufgetreten war und welche, wie oben wiederholt erwähnt wurde, von ähnlichen Riesenzellen umschlossen waren, Blutpigment.

Mastzellen waren überall im Gewebe sehr häufig zu finden.

Schließlich sei noch erwähnt, daß wir injiziertes Jodöl außer in den oben erwähnten Hohlräumen und zelligen Infiltrationsherden noch besonders in der Umgebung der neugebildeten Gefäße und in der Nähe der Infiltrationsherde und überall im neuen zelligen Bindegewebe zwischen den Muskelfasern fanden; ebenda fanden sich gleichzeitig neugebildete Gefäße.

Wie ist die Entstehung und das Verhalten dieser $\mathrm{Hohl}$ $\mathrm{räume} z \mathfrak{m}$ erklären? Wolters spricht in seiner zweiten Arbeit die Ansicht aus, daß die Hohlräume leere Sarkolemmschläuche seien, welche nach der auf chemischem Wege erfolgten Zerstörung von Muskelfasern zurückblieben und daß dann von dem die Hohlräume auskleidenden Sarkolemm eine Wucherung ausgehe, welche zur Bildung von Scheidewänden und so zur Umwandlung ursprünglich großer Räume in mebrere kleinere führe. In seiner ersten Arbeit hatte er dargestellt, daß die Hoblräume einen starren Kontur zeigten, häufig untereinander konfluierten und oft mit Endothel ausgekleidet wären. weshalb er sie als Lymphgefäße auffaßte.

Nach Pezzoli ließen einzelne größere Räume sogar eine eigene Wandung erkennen, deshalb glaubt er, daß sie nicht Sarkolemmaschläuche, sondern Lymphräume darstellen müßten.

Nach meinem Dafürhalten handelte es sich in unserem Falle nicht um präformierte Hohlräume, sondern die letzteren dürften dadurch. zu stande gekommen sein, daß die Gewebsbestandteile durch die Injektionen mechanisch auseinandergedrängt wurden. Unter dem reizenden Einfluß der in den so entstandenen Gewebsspalten enthaltenen Injektionsmassen kam es zu einer reaktiven Entzündung.

Es entwickelt sich ein Granulationsgewebe, das zahlreiche vielkernige Riesenzellen enthielt, die der Oberfläche des öligen Inhalts der Hohlräume anlagen. Dieses zahlreiche Granula- 
tionsgewebe schrumpft schließlich und verwandelt sich in kernarmes Bindegewebe, in dem Hohlräume, die öltropfen einschließen, enthalten sind. Die zellige Auskleidung der letzteren stammt von dem Granulationsgewebe her.

Über die Herkunft und Abstammung der Fremdk $\mathrm{k}$ r per-Riesenzellen, welche wir außerhalb der Hohlräumewände hie und da im Gewebe und zwar in den starken Infiltrationsherden teils einzeln, teils in kleinen Gruppen, besonders in den kleinen mit Jodöl gefüllten Gewebsspalten finden, lassen sich wegen der weit vorgeschrittenen Entwicklung der entzündlichen Vorgänge sichere Anhaltspunkte nicht gewinnen, doch möchte ich mit Rücksicht auf einige von mir gemachte Beobachtungen es nicht für unwahrscheinlich halten, dak an ibrer Genese sowohl Lymphocyten (Ziegler) als auch fixe Bindegewebszellen beteiligt waren.

Auf Grund der oben erwähnten Veränderungen, welche die Einspritzungen von schwefelhaltigem Jodöl ergeben hatten, schien es mit Rücksicht darauf, daß die gefundenen Veränderungen in mancher Hinsicht syphilitischen Gewebsveränderungen, wie oben erwähnt, glichen, wichtig festzustellen, ob an den Injektionsstellen Gummabildung stattgefunden habe oder nicht; im letzten Falle, d. h. wenn diese Gewebsveränderungen gänzlich durch die Medikamentinjektionen entstanden waren, tauchte die weitere Frage auf, ob die Hauptrolle bei diesen Veränderungen dem Hauptbestandteil dieses Medikaments zufiel, oder ob diese Veränderungen auch bloß durch das gleichzeitig benutzte Sesamöl verursacht waren.

Ich wählte zu diesem Zwecke den Weg des Tierexperimentes, indem ich zum Teil mit diesem Medikament, zum Teil mit bloßem Sesamöl experimentierte. Ich habe mich wesentlich auf 4 Kaninchen und 2 Ratten beschränkt und bei jedem Kaninchen mittelst einer Prarazschen Spritze je $1 g$ 10\% iges Jodöl und Sesamöl in das subkutane und intramuskuläre Gewebe beider Riückenseiten eingespritzt und die gesetzten Veränderungen nach verschieden langer Dauer (1 bis 28 Tage) untersucht. Dabei habe ich zwischen subkutanen und intramuskulären Injektionen keinen bemerkbaren Unterschied bemerkt. Auch hatte ich wegen zu großer Dosen bei 2 Kaninchen kein sehr gutes Resultat. Später habe ich daher bei 2 Ratten sehr vorsichtig bloß $1-2$ Tropfen sublutan auf beiden Rückseiten und zwar links Jodöl und rechts Sesamöl injiziert und die eine Ratte 3 Tage nach der Injektion, die andere 9 Tage nachher getötet und die mit der Haut und den Muskeln 
herausgeschnittenen Gewebsstücke der injizierten Stellen zur Untersuchung benutzt.

Es ergab sich dabei, daß bei Tieren durch das Jodöl genau dieselben Veränderungen erzielt worden waren, wie sie bei unserem Patienten zur Beobachtung kamen, insbesondere fanden sich reichlich jene Herde wieder, die miliaren Tuberkeln oder Gummen frappant ähnlich waren. Ich verzichte auf eine genaue Schilderung der histologischen Veränderungen, da ich dabei nur das wiederholen müßte, was ich oben über die beim Menschen gefundenen Veränderungen gesagt habe. Bei Injektionen von Sesamöl allein traten stärkere entzündliche Veränderungen nicht auf.

Wenn ich nun das Ergebnis meiner Untersuchungen am Menschen und an Tieren noch einmal kurz zusammenfasse und dasselbe mit den analogen Untersuchungen anderer Autoren mit unlöslichen Quecksilberpräparaten vergleiche, so mulf ich sagen, daß die Veränderungen, welche nach der Injektion von schwefelhaltigem Jodöl in das subkutane Gewebe entstehen, hauptsächlich als Entzündung aufzufassen sind, ebenso wie andere Autoren verschiedene Entzündungsgrade von der Art der Quecksilberpräparate abhängig sein lassen.

Diesbezügliche Untersuchungen sind schon mehrfach in der Literatur niedergelegt. Besonders hervorzuheben sind die experimentellen Untersuchungen von Jadassohn, welcher an Kaninchen mit Hg-Salizyl und $\mathrm{Hg}$-Thymolacet experimentierte. Doch können die beim Tiere erhaltenen Resultate, nach Jadassohns eigenen Worten, nicht ohne weiteres die beim Menschen hervorgerufenen Veränderungen erklären, besonders deshalb, weil die Muskulatur der Tiere den mechanischen und chemischen Einwirkungen des injizierten Präparates nicht genügend Widerstand leistet.

Bezüglich der Befunde am Menschen kennen wir noch am besten die durch das OI. ciner. und das Kalomel, teils im subkutanen, teils im Muskelgewebe hervorgerufenen Veränderungen. Wenn wir die älteren zum Teil mangelhaften, von Wolters in seiner Monographie erwähnten Untersuchungen bei Seite lassen, so müssen namentlich die exakten Untersuchungen von Jullien, Allgeyer und A udry besonders hervorgehoben werden, da sie uns ein ziemlich klares Bild von den durch Kalomel im Muskelgewebe erzeugten Veränderungen geben. Das Kalomel besitzt danach größere irritative Eigenschaften als das Ho-Salizyl., denn sowohl A l lgeyer als Jullien und A udry sprechen von Höhlen, deren Wandungen mit Detritusmassen bedeckt und die mit eiterähnlicher Flüssigkeit gefüllt war. Auch in unserem Falle fanden sich solche allerdings nicht mit Eiter, sondern mit Öl erfüllte - Hohlräume.

Wenn wir nun die durch unser Jodöl erzeugten lokalen Veränderungen ungefähr den Reizungserscheinungen gleich erachten, die durch das meist verwendete $\mathrm{Hg}$-Salizyl hervorgebracht werden und uns erinnern, daß von einigen Autoren Gummata an alten Injektionsstellen beobachtet worden sind, so mïssen wir auch die Frage aufwerfen, ob vielleicht auch an den Injektionsstellen von Jodöl Gummabildung auftritt oder nicht. 
Was das Auftreten von Gummen an den Injektionsstellen von $\mathrm{Hg}$ Salizyl betrifft, so berichtete als der erste $\mathrm{Kulisch}(\mathbf{2 5})$ auf dem VII. Kongreb der Devtschen dermatologischen Gesellschaft zu Breslau und in der dermatologischen Sektion der 73. Versammlung deutscher Naturforscher und Ärzte zu Hamburg, später in einer ausführlichen Mitteilung. über gummöse Bildung an alten Injektionsstellen von Salicylquecksilber. Er hebt aus der Krankengeschichte eines seiner Pat. die bisher nicht registrierte auffallende Tatsache hervor, daß nach Verlauf von 5 Jahren noch Reste der früheren Quecksilberivjektionen auf chemischem Wege im Gewebe nachgewiesen werden konnten und geht dann unter Würdigung der einzelnen Momente näher auf die Begründung der Diagnose "Gumma“ ein. Bei dieser Diagnose wird das Hauptgewicht auf die wiederholte günstige Beeinflussung des Krankheitsprozesses durch eine spezifische Behandlung gelegt.

Kurz nach Kulis ch teilte Schmidt (26) die Krankengeschichte eines Patienten mit, bei dem 10 Jahre nach der Injektion Gummabildung im AnschluB an einen Unfall auftrat.

Werner (27) stellte im ärztlichen Verein zu Hamburg 2 Kranke vor, bei denen Gummabildung aufgetreten war und zwar bei dem einen 4 Tage, bei dem anderen 6 Jahre nach der Injektion.

In neuester Zeit veröffentlicht Juliusberg (28) die Geschichte dreier Patienten, bei denen bezw. $11 / 8,3 / 4$ und $1-6$ Jahre zwischen Injektion von Hg-Thym. acet. und Gummabildung lagen.

Für unsere Frage sind die von den oben genannten Autoren beschriebenen Fälle nicht zu verwerten, da zur histologischen Untersuchung Material nicht zur Verfügung stand. Aus der Mitteilung von $\mathrm{Kulisch}$ der im Zentrum Detritus und metamorphosierte Zellen fand, lassen sich in differentialdiagnostischer Beziehung ebenfalls keine Schlüsse ziehen. In dem von Chotzen (29) untersuchten Falle, der tine 6 Jahre nach stattgehabter Injektion von Hg-Thym. acet. zurückgebliebene Geschwulst. betrifft, bandelt es sich, da hier nicht eine frisch aufgetretene, sondern seit 6 Jahren bestehende Bildung vorliegt, wohl unzweifelhaft um ein abgelsapseltes altes $\mathrm{Hg}$-Infiltrat.

Die Ansichten über die Entstehung von Gummata an Injektionsstellen von Quecksilberpräparaten befinden sich also noch im Stadium hypothetis ${ }^{2}$ er Anschauungen. Die Meinungen gehen darüber auseinander, ob diese Knotenbildungen in der Tat mit Sicherheit als Gummen aufzufassen sind, oder ob sie vielleicht, wie dies Chotzen auf der Hamburger Naturforscherversammlung gemeint hat, nur erweichte chronische, durch Quecksilberabkapselung veranlaßte Infiltrate darstellten, oder ob endlich entsprechend der auf der Hamburger Naturforscherversammlung geäußerten Vermutung von $\mathrm{Hahn}$ und Mense etwaige Quecksilberreste an diesen Stellen durch Joddarreichung dio Bildung ätzenden Quecksilberjodids verursacht und damit eine Reizung und Erweichung des umgebenden Gewebes veranlaßt haben. Die letzte Annabme wird schon durch die Tatsache hinlänglich widerlegt, daß von $\mathrm{Juliusberg}$ obigen drei Patienten, deren ganze Syphilisbehandlung unter Aufsicht der Klinik stattfand, zwei vor der Knotenbildung Jod in keiner Form erhalten hatten.

Fr. L esser (15) hat durch einwandfreie Experimente dargetan, daß es bei vorhandenen Depots, die von Salizyl- und Thymolquecksilber herrühren, auch bei reichlicher Joddarreichung vicht zur Bildung des ätzenden $Q$ u ecksil ber-Jodid kommt. Demnach können wir heute noch nicht an die Entstehung von Gummabildung an alten Injektionsstellen von unlöstichem Quecksilber glauben, bis sichere deutliche histologische Untersuchungen vorliegen. Da obige Mitteilungen bezüglich der klinischen Erscheinungen besonders des zeitlichen Auftretens von Gummabildung zweifelhaft sind, so müssen wir diese Knotenbildung 
wahrscheinlich nur den unlöslichen Hg-Injektionen zuschreiben und verweisen auf unsere Tierexperimente und die dabei erhobenen und oben beschriebenen histologischen Befunde.

Am Ende meiner Arbeit möchte ich darauf hinweisen. daß der vorliegende Sektionsbefund beweist, wie langsam das Jod aus subkutanen Jodölinjektionen resorbiert wird und daß man noch nach 4 Monaten beträchtliche Depots an den Injektionsstellen findet. Daraus geht hervor, daß man für die Therapie nicht allzugroße Hoffnungen an dieselben knüpfen darf; das besonders, wo Gefuhr im Verzug ist, Jodipininjektionen kontraindiziert sind.

Die langsame Resorption subkutaner Öldepots ist von vielen Autoren bestätigt worden.

Winternitz hat an Tieren und Menschen die langsame Resorption subkutaner Öldepots gezeigt und nebenbei dargetan, dah der Jodzusatz die Resorption des ôles nicht ungünstig beeinflußt, weder ein $10 \%$ iger noch ein $1 \%$ iger.

Er wies nach, daß höchstens $2 g$ Fett pro Tag, in den ersten 8 Tagen weniger, resorbiert werden; daß die Resorption von $500 \mathrm{~g}$ daher Monate braucht, daß infolgedessen diese Methode zur subkutanen Ernährung nicht in Frage kommen kann. Bei seinem Sektionsbefunde ist das wesentliche der Umstand, daß nach 3-4. Monaten nach der letzten Injektion in der Lumbalgegend, in größerer Ausdehnung, unter der Haut und in den Muskelinterstitien sich rahmähnliche ölreste fanden und daß dieselben, ausgequetscht and ehemisch analysiert, noch den ursprünglichen Jodprozentgehalt hatten. Für Winternitz unwesentlich, aber für meine Betrachtungen nicht unwichtig ist, daß nirgends bei seinen Sektionen ein Infiltrat an den Injektionsstellen erwähnt wird.

Es war sonach der Jodgehalt des nicht resorbierten Fettes völlig intakt geblieben und der rahmartige emulgierte Zustand vielleicht im wesentlichen rein mechanisch durch die Fortbewegung unter der Hant und in den Muskelinterstitien zu stande gekommen, jedenfalls ohne Abspaltung von Jod.

Außerdem konnte bei seinen Versuchen im Blute der Patienten Jod nicht sicher, höchstens minimalste Jodspuren, die praktisch nicht in Betracht kommen können, nachgewiesen werden. Dieser negative Blutbefund läßt vermuten, $\alpha a ß$ in den entfernter liegenden Organen (Leber, Knochenmark, Mesenterialfett u. a.) Jodfette nur in minimalen Mengen oder gar nicht vorhanden sem können, dagegen fanden sich Jodspuren in der betreffenden Muskulatur. Die Jodausscheidung verhält sich aber nach Injektion von Jodfetten ebenso wie bei der Aufnahme der Jodalkalien per os. Das Jod wird sehr rasch, innerhalb 24 Stunden, von gesunden Nieren wieder ausgeschieden. Von Jodfetten kommt noch in Betracht, daß ein in Unterernährung befindlicher Organismus mehr davon oxydiert als ein gut genährter.

Feibes (10) hat 402 Tage nach der letzten Injektion Jod im Urin gefunden; Welander (7), Möller (9), und Fr. Lesser (11) haben auch die langsame Resorption des Jods von subkutanen Jodipininjektionen festgestellt and bemerkt, daß es bezüglich des Grades der Wirksamkeit nicht gleichwertig mit Jodkali ist, wobei sie ihre Nichtgiftigkeit und jegliches Fehlen von Nebenwirkung konstatieren konnten. Trotzdem hält $W$ elander das Jodipin in Form von subkutanen Injektionen für das einzige Mittel, welches in bequemer Weise eine 
gleichmäßige, chronische Jodmedikation ohne unangenehme Nebenwirkungen ermöglicht, die in prophylaktischer Hinsicht für spätere Tertiärbzw. parasyphilitische Erkrankungen von großer Bedeutung sein kann.

Im Dresdner pathologischen Institut des Herrn Professor Dr. Schmorl war zweimal Gelegenheit, bei Sektionen noch monatelang nach der letzten Jodipininjektion erhebliche rahmähnliche Depots in der Nähe der Injektionsstelle zu sehen.

Wenn wir nun sehen, daß weder Öl an sich noch ein Jodzusatz wie im Jodipin eine starke entzündliche Reizung zu verursachen pflegen, so müssen in unserem Falle besondere Entzündungsreize gewirkt haben. Daß es bei unserem Kranken um Gum a a bildung sich gehandelt habe, können wir nicht annehmen, da die gleichen histologischen Veränderungen bei nichtsyphilitischen Tieren durch die gleichen Injektionen hervorgerufen wurden.

Da sich unser Jodöl durch den Schwefelgehalt rom Jodipin unterscheidet, ist in diesem der Entzündungserreger zu suchen. Aber nicht in allen Fällen, die subkutane Injektionen dieses Jodschwefelöles erhielten, waren - wenigstens klinisch nicht Infiltrate zu bemerken. Ich vermute, daß die Schwefelbindung nicht beständig ist und die bei unserem Patienten und den Versuchstieren benutzte Lieferung zersetzt war. Vielleicht ist Schwefelwasserstoff der Erreger der beschriebenen Entzündung gewesen, wie wir nach den Injektionen unlöslicher $\mathrm{Hg}$-Verbindungen das Sublimat als solchen annehmen. Eine genaue chemische Untersuchung hat nicht stattgefunden.

Das Resumé meiner Untersuchungen lautet:

1. Bei den sublutanen 'Injektionen von schwefelhaltigem Jodöl bemerkten wir im Gegensatze zu gewöhnlichen öl, spez. Sesamöl, starke Reizerscheinungen in dem Gewebe.

2. Durch die mechanische Wirkung der eingespritzten Masse bilden sich einfache oder konfluierende Hohlräume in den Gewebsspalten; die Masse wird durch die Gewebsspalten noch weiter nach der Peripherie getrieben, wo verschiedene kleine Hohlräumchen sich bilden und noch weiter durch die Gewebselemente, zwischen denen wir sie als Öltröpfchen finden

3. Um Fettropfen bildet sich bereits nach 3 Tagen eine starke Entzündung und dabei treten Zellen auf, die ihrem morphologischen Charakter nach als Leukozyten und Abkömmlinge fixer Gewebszellen anzusehen sind (Granulationsgewebe). Ferner wurden Riesenzellenbildungen beobachtet. Die zellige Wucherung zerlegt die größeren und kleineren Fettropfen, wobei eine reichliche Aufnahme von Fettröpfchen in die Zellen stattfindet. Stellenweise kommt es zur Bildung von Wucherungs- 
berden, welche miliaren Gummen oder Tuberkeln sehr ähnlich sind. Die zentrale Nekrose in solchen Herden entsteht unter dem direkten schädigenden Einfluß des schwefelhaltigen Jodöls, welches auch eine Nekrose der Muskelfasern hervorzurufen vermag.

4. Das Granulationsgewebe schrumpft allmählich und verwandelt sich in Bindegewebe.

5. Das subkutan injizierte schwefelhaltige Jodöl wird langsam in einigen Monaten und zwar vorwiegend durch Zellen, resorbiert, aber schneller als Sesamöl, denn es findet infolge der starken Reizung der Zellen eine lebbaftere Phagocytose statt.

6. Dem Reiz des schwefelhaltigen Jodöls gleich erachten wir den durch Salizylquecksilber. An der Gummabildung durch letzteres zweifeln wir. Unsere Kontrollversuche mit Jodöl an Ratten berechtigen dazu.

7. Für die Zwecke der Ernährung hat die subkutane Jodfettinjektion keinen Wert. Für die 'Therapie der Syphilis nur einen bedingten.

Zum Schlusse gestatte ich mir, den von mir hochverehrten Herren Obermedizinalrat Prof. Dr. Schmorl und Oberarzt Dr. Werther für die Anregung za der vorliegenden Arbeit und die freundliche Unterstützung bei der Durchsicht der histologischen Präparate meinen verbindlichsten Dank auszusprechen.

\section{Literatur.}

1. Dtsch. med. Woch. 1897. Nr. 23, Hoppe-S eylers Zeitschrift für physiologische Chemie. 1898. XXIV. H. 5/6 u. München. med. Woch. 1903. Nr. 59. p. 1241.

2. Berl, klin. Woch. 1899. Nr. 25 u. Dtsoh. med. Woch. 1900. Nr. 26.

3. Fortschritte d. Medizin. 1899. Bd. XVII. Nr. 46.

4. Protokoll d. Moskauer venerolog. Gesellschaft 1899 bis 1900. Bd. IX. p. 142 .

5. Beiträge z. Dermat. u. Syph, Festschr. 1900.

6. Korrespond. Blätter der Allgem. ärztlichen Ver. von Thüringen. 1901. Nr. 6 u. 7.

7. Therap. Monatsh. Juni, 1901.

8. Arch. f. Derm. u. Syph. 1901. Bd. LVII. p. 63.

9. ib. p. 101 .

10. ib. p. 113.

11. Derm. Zeitschr. 1902. Bd. IX.

12. Arch. f. Derm. u. Syph. 1903. Bd. LXIV. p. 91.

13. Ref. Münch. med. Woch. 1903. Nr. 13. p. 588.

14. ib. 1903. Nr. 38. p. 1634. 
15. Dtsch. med. Woch. 1896. Nr. 30.

16. Arch. f. Derm. u. Syph. 1888. Bd. XV. p. 782.

17. Dtsch. med. Woch. 1901 . Nr. 47 u. 48 u. Arch. f. Derm. u. Syph. 1902. Bd. LX. p. 91.

18. Arch. f. Derm. u. Syph. 1895. Bd. XXXII. p. 149 u. ib. 1897. Bd. XXXIX. p. 163.

19. Wien. klin. Woch. 1902. Nr. 51. p. 1360.

20. Ann. de Dermat. et Syphiligr. 1896. p. 171 u. Archives générales de medicine (Mai 1890).

21. Arch. f. Derm. u. Syph. 1901. Bd. LV. p. 1.

22. Journal des maladies entanées et syphilitiques. 1901. H. 6.

23. Virchows Areh. 1880. Bd. LXXX. p. 281.

24. Ref. Arch. f. Derm. u. Syph. 1902. Bd. LXI. p. 408.

25. Monatsschr. f. Unfallheilk. 1901. Nr. 8.

26. Ref. Dtsch. med. Woch. 1902. Nr. 12. p. 92.

27. Münch. med. Woch. 1902. Nr. 15. p. 645.

28. Ref. Dtsch. f. Derm. u. Syph. 1902. Bd. LXI. p. 420.

\section{Erklärung der Abbildungen auf Taf. XII.}

Fig. 1. Querschnitte im mittleren Teil des Stranges. Fixierung mit Formalinlösung, Schneiden mit Gefriermikrotom Färbung mit Sudan III, Hämatoxylin, in Glyzerin eingeschlossen. Vergr. Zeiß Ok. $3 \cdot 0,0 b j . A A .-$ a) Hohlräume, das durch Sudan III rotgefärbte Jodöl enthaltend. Sie sind ausgekleidet mit abgeplatteten Zellen, die der Oberfläche des öls anliegen. - b) Konfluierenden Honigwaben ähnliche Hohlräume, die von konfluierenden Infiltrationsherden umgeben sind. c) Infiltrationsherde, meist in den Umgebungen der Gefäße, in den Herden kleine Öltröpfehen. - d) Homogen veränderte fibrinöse Exsudationsbezirke, in ihrer Umgebung versohieden große und kleine Öltröpfchen; davon feinste Fettröpfchen und Fettkörnchen in den Riesenzellen, fixen Gewebszellen und Leukocyten anfgenommen. - e) GefäB mit bindegewebiger Verdickung der Wand und Wucherung der Endothelien. f) Neugebildete Gefäße mit den in ibrer Umgebung frei gelegenen Öltröpfchen. $-g$ ) Zwischen einzelnen Muskelfasern Bindegewebsbildung mit gefäßreicher zelliger Infiltration.

Fig. 2. Feinste Fettkörnchen enthaltende Fremdkör perriesenzellen ausden mittlerenLängs-und Querschnitten des Stranges:

Fixierung und Färbung wie Figur 1. Vergr. Zeiß. Ok. 1, Obj. Immersion. $1 / 12(420 \mathrm{x})$.

In den Fremdkörperriesenzellen, sowie in den benachbarten Gewebszellen und Leukocyten feinste Fettkörnchen. 


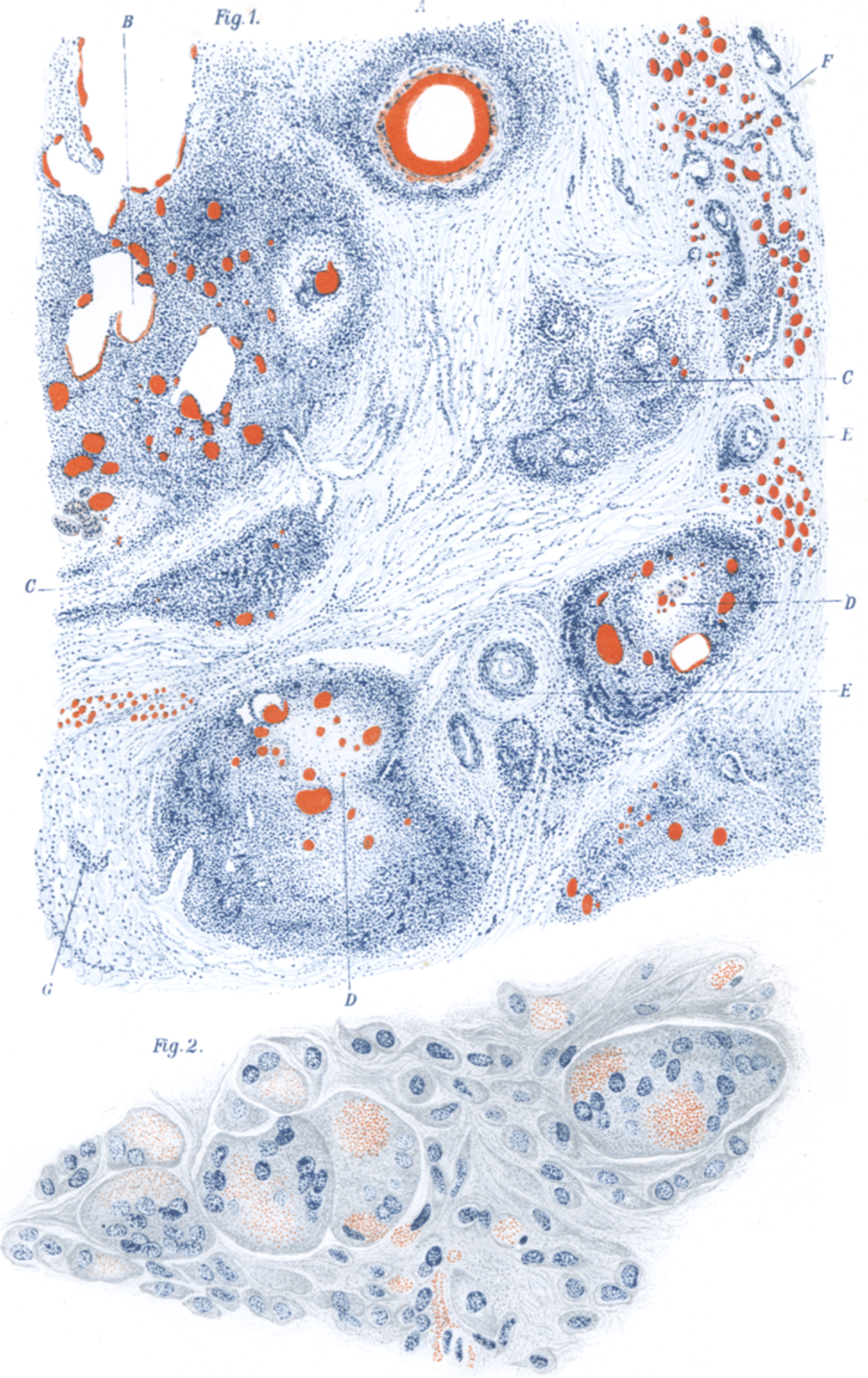

Tokujiro Suzuki : lokale Verinderung nach hypodemin. Jnj. 